DOI: $10.3724 /$ SP.J.1218.2012.00329

\title{
欠驱动自主水面船的非线性路径跟踪控制
}

\author{
高 剑, 刘富樯, 赵 江, 严卫生 \\ (西北工业大学航海学院, 陕西 西安 710072)
}

\begin{abstract}
摘 要: 基于级联方法提出一种欠驱动自主水面船的全局 $\mathcal{K}$ 指数稳定路径跟踪控制算法. 采用以自由路径参 考点为原点的 Serret-Frenet 坐标系建立路径跟踪误差的动态模型, 以路径参数的变化率为附加控制输入, 克服了以 正交投影点为坐标原点时的奇异值问题. 设计路径跟踪航向角指令, 将路径跟踪模型分解为位置跟踪子系统和航向 角、前向速度跟踪子系统两个子系统级联的形式, 设计航向角和前向速度的全局指数稳定跟踪控制器, 应用级联系 统理论证明了路径跟踪误差的全局 $\mathcal{K}$ 指数稳定性. 数学仿真和自主水面船湖上实验结果验证了该路径跟踪控制算 法的有效性.
\end{abstract}

关键词: 自主水面船; 欠驱动; 路径跟踪控制; 级联方法

中图分类号: TP24 文献标识码: A 文章编号: 1002-0446(2012)-03-0329-08

\section{Non-linear Path Following Control of Underactuated Autonomous Surface Vehicles}

\author{
GAO Jian, LIU Fuqiang, ZHAO Jiang, YAN Weisheng \\ (College of Marine Engineering, Northwestern Polytechnical University, Xi'an 710072, China)
}

\begin{abstract}
The globally $\mathcal{K}$-exponentially stable path following control of an underactuated autonomous surface vehicle (ASV) is proposed based on the cascaded approach. The dynamics model of the ASV path following errors is derived utilizing the Serret-Frenet coordinate originated at the free path reference point, which creates an added control input, i.e., the change rate of the path parameter, to avoid the singularity problem when adopting the coordinate originating at the orthogonal projection point. The path following reference yaw is designed, and the whole path following model is decomposed into two cascaded sub-models, named the position tracking sub-model and the yaw angle and surge velocity tracking sub-model. The global $\mathcal{K}$-exponential stability of the path following errors is proved using the cascaded system theory with the globally exponentially stable yaw angle and surge velocity tracking controllers. The results of the mathematic simulations and the lake trials of a real ASV demonstrate the performance of the proposed path following control algorithm.
\end{abstract}

Keywords: autonomous surface vehicle; underactuated; path following control; cascaded approach

\section{1 引言 (Introduction)}

不具备侧向推进器的自主水面船 (autonomous surface vehicle, ASV) 是一类典型的欠驱动系统 ${ }^{[1]}$, 它必须通过偏航力矩和推力 2 个独立输入控制平面 坐标和航向角 3 个运动自由度. 路径跟踪控制 (path following control) 是欠驱动 ASV 运动控制领域研究 的热点之一, 它是指给定平面内一条与时间无关的 曲线路径, ASV 从任意初始状态出发, 收玫于该曲 线并沿该曲线以期望前向速度运动.

Serret-Frenet 法是一种最为常用的路径跟踪控 制方法, 它以 ASV 在参考路径上的正交投影点为原 点, 以路径的切线方向为 $x$ 轴建立 Serret-Frenet 坐 标系, 用 ASV 在该坐标系下的法向距离描述路径跟 踪误差 ${ }^{[2]}$. 当 ASV 到投影点的距离为该点的曲率半
径时, 投影点的移动速度为无穷大, 成为路径跟踪 控制中的奇异点, 因此 ASV 到路径的距离不能大于 路径的最小曲率半径, 无法获得全局解.

法国蒙彼利埃机器人研究所的 Lapierre ${ }^{[3-4]}$ 提出 以路径上的自由点为参考点, 建立 Serret-Frenet 坐 标系, 并以曲线参数变化率 $\dot{\varpi}$ 为附加控制量, 使 ASV 在 Serret-Frenet 坐标系中的切向误差渐近稳 定，从而克服了奇异值问题. 该算法存在 2 个问题: (1) 采用反步法 (backstepping) 设计偏航力矩结果较 为繁琐; (2) 控制律中包含侧滑角的二次导数, 需对 前向控制力求导, 且当前向速度为 0 时侧滑角无定 义.

此外，挪威科技大学的 Breivik 基于导引方法 研究了路径跟踪控制问题 ${ }^{[5]}$, 采用与 Lapierre 相似 
的路径参考坐标系, 并基于运动学模型获得了参 考点速度和航向指令, 证明了系统的全局渐近稳定 性, 但未考虑速度控制和航向控制问题. 葡萄牙 IST 的 Maurya 提出了内环一外环直线路径跟踪控制方 法 ${ }^{6]}$, 以路径跟踪为外环, 航向角跟踪控制为内环, 并采用输入输出稳定性理论和小增益理论证明了直 线路径跟踪的稳定性. 该算法的优点是运动学和动 力学控制解耦, 易于算法设计和工程实现, 但未证 明一般曲线路径跟踪的稳定性.

本文在 Lapierre 方法的基础上采用级联方 法 ${ }^{[7-8]}$ 研究 ASV 的曲线路径跟踪控制问题, 并进 一步证明了路径跟踪误差的全局 $\mathcal{K}$ 指数稳定性. 该 算法具有 Maurya 提出的内环一外环控制结构的特 点, 并基于速度指令定义侧滑角, 解决了对控制力 求导和侧滑角无定义的问题. 最后采用挪威科技大 学的 CybershipII ASV 数学模型进行仿真研究, 并完 成了 ASV 湖上实验, 验证了该控制算法的有效性.

\section{2 问题描述（Problem formulation）}

根据文 [9], 欠驱动 ASV 的运动模型为

$$
\begin{aligned}
& \dot{x}=u \cos \psi-v \sin \psi \\
& \dot{y}=u \sin \psi+v \cos \psi \\
& \dot{\psi}=r
\end{aligned}
$$

和

$$
\begin{aligned}
& \dot{u}=\frac{m_{22}}{m_{11}} v r-\frac{d_{11}}{m_{11}} u+\frac{1}{m_{11}} X \\
& \dot{v}=-\frac{m_{11}}{m_{22}} u r-\frac{d_{22}}{m_{22}} v \\
& \dot{r}=\frac{m_{11}-m_{22}}{m_{33}} u v-\frac{d_{33}}{m_{33}} r+\frac{1}{m_{33}} N
\end{aligned}
$$

式中, $(x, y)$ 为 ASV 船体坐标系 $\{B\}$ 的原点 $O$ 在全 局坐标系 $\{N\}$ 下的坐标, $\psi$ 为航向角, $u$ 为前向速 度, $v$ 为侧向速度, $r$ 为航向角速度, 参数 $m_{11}=m-$ $X_{\dot{u}}, m_{22}=m-Y_{\dot{v}} 、 m_{33}=I_{z z}-N_{\dot{r}}, d_{11}=-X_{u} 、 d_{22}=$ $-Y_{v} 、 d_{33}=-N_{r}, m$ 为 $\mathrm{ASV}$ 质量, $I_{z z}$ 为偏航转动惯 量, $X_{\dot{u}} 、 Y_{\dot{v}} 、 N_{\dot{r}}$ 为附加质量, $X_{u} 、 Y_{v} 、 N_{r}$ 为线性阻尼 系数, $X$ 为前向推力, $N$ 为偏航力矩.

如图 1 所示, $\Omega$ 为平面内的一条曲线路径, 由 曲线参数 $\varpi$ 描述, $P$ 为 $\mathrm{ASV}$ 的路径跟踪参考点, 以 $P$ 为原点建立 Serret-Frenet 坐标系 $\{S F\}$, 其中 $x_{\mathrm{sf}}$ 轴 沿路径在 $P$ 点的切线方向, $y_{\mathrm{sf}}$ 轴沿 $P$ 点的法线方 向, $z_{\mathrm{sf}}$ 轴向下构成右手坐标系. ASV 路径跟踪误差 由点 $O$ 在 $\{S F\}$ 坐标系中的坐标 $\left(\tau_{\mathrm{e}}, n_{\mathrm{e}}\right)$ 定义.

点 $P$ 坐标为 $\left(x_{\mathrm{p}}(\varpi), y_{\mathrm{p}}(\varpi)\right)$, 由曲线参数 $\varpi$ 唯 一确定, 坐标对路径参数的一阶和二阶导数表示为

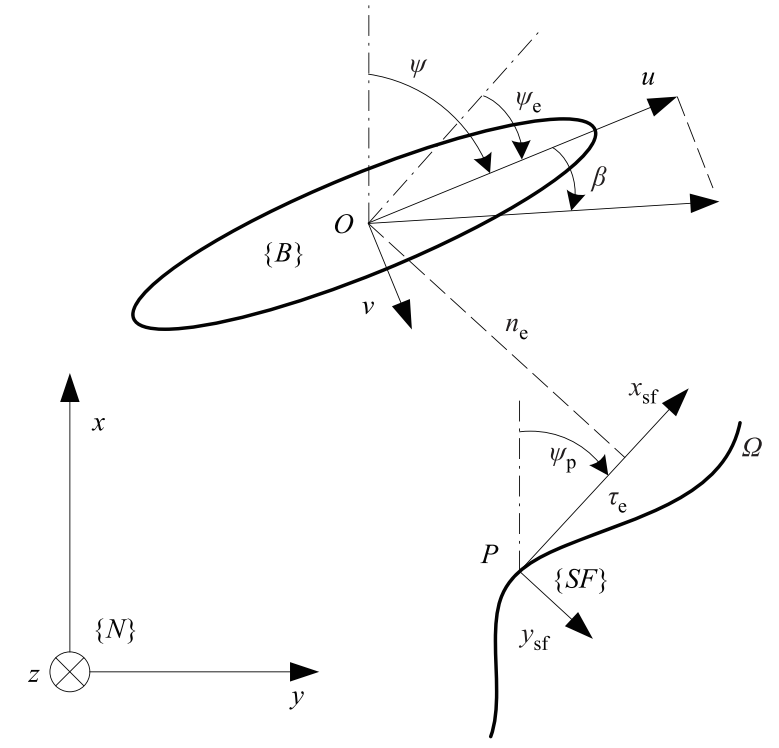

图 1 Serret-Frenet 坐标系及路径跟踪误差

Fig.1 Serret-Frenet coordinate frame and path following errors

$$
\begin{array}{ll}
x_{\mathrm{p}}^{\prime}(\varpi)=\frac{\mathrm{d} x_{\mathrm{p}}}{\mathrm{d} \Phi}, & y_{\mathrm{p}}^{\prime}(\varpi)=\frac{\mathrm{d} y_{\mathrm{p}}}{\mathrm{d} \varpi} \\
x_{\mathrm{p}}^{\prime \prime}(\varpi)=\frac{\mathrm{d}^{2} x_{\mathrm{p}}}{\mathrm{d} \varpi^{2}}, & y_{\mathrm{p}}^{\prime \prime}(\varpi)=\frac{\mathrm{d}^{2} y_{\mathrm{p}}}{\mathrm{d} \varpi^{2}}
\end{array}
$$

点 $P$ 沿曲线运动的速度可以表示为

$$
U_{\mathrm{p}}=\dot{\varpi} \sqrt{{x_{\mathrm{p}}^{\prime 2}}^{2}(\varpi)+{y_{\mathrm{p}}^{\prime}}^{2}(\varpi)}
$$

点 $P$ 切向量的方位角, 即 $x_{\mathrm{sf}}$ 轴与 $x$ 轴的夹角为

$$
\psi_{\mathrm{p}}(\varpi)=\arctan \frac{y_{\mathrm{p}}^{\prime}(\varpi)}{x_{\mathrm{p}}^{\prime}(\varpi)}
$$

其角速率为

$$
\begin{aligned}
r_{\mathrm{p}}(\varpi) & =\frac{\partial \psi_{\mathrm{p}}}{\partial \varpi} \dot{\varpi} \\
& =\frac{x_{\mathrm{p}}^{\prime}(\varpi) y_{\mathrm{p}}^{\prime \prime}(\varpi)-y_{\mathrm{p}}^{\prime}(\varpi) x_{\mathrm{p}}^{\prime \prime}(\varpi)}{x_{\mathrm{p}}^{\prime 2}(\varpi)+y_{\mathrm{p}}^{\prime 2}(\varpi)} \dot{\varpi}
\end{aligned}
$$

ASV 路径跟踪误差模型 ${ }^{[3-4]}$ 可表示为

$$
\begin{aligned}
& \dot{\tau}_{\mathrm{e}}=-U_{\mathrm{p}}+r_{\mathrm{p}} n_{\mathrm{e}}+u \cos \psi_{\mathrm{e}}-v \sin \psi_{\mathrm{e}} \\
& \dot{n}_{\mathrm{e}}=-r_{\mathrm{p}} \tau_{\mathrm{e}}+u \sin \psi_{\mathrm{e}}+v \cos \psi_{\mathrm{e}} \\
& \dot{\psi}_{\mathrm{e}}=r-r_{\mathrm{p}}
\end{aligned}
$$

其中, $\psi_{\mathrm{e}}=\psi-\psi_{\mathrm{p}}$.

欠驱动 ASV 路径跟踪控制的目标为, 给定由 参数 $\varpi$ 描述的参考路径 $\Omega$ 和有界前向速度指令 $u_{\mathrm{d}}(t)$, ASV 从任意的初始位置和航向角出发, 寻找 前向推力 $X$ 、偏航力矩 $N$ 和曲线参数的变化率 $\dot{\varpi}$, 使 ASV 路径跟踪误差 $\left(\tau_{\mathrm{e}}, n_{\mathrm{e}}\right)$ 全局渐近稳定, 前向 速度 $u$ 渐近收敛于期望速度 $u_{\mathrm{d}}$. 


\section{3 基于级联方法的 ASV 路径跟踪控制 (ASV} path following control based on the cascaded approach )

定义速度跟踪误差

$$
u_{\mathrm{e}}=u-u_{\mathrm{d}}
$$

将路径跟踪误差模型式 (6) 重写为

$$
\begin{aligned}
& \dot{\tau}_{\mathrm{e}}=-U_{\mathrm{p}}+r_{\mathrm{p}} n_{\mathrm{e}}+u_{\mathrm{d}} \cos \psi_{\mathrm{e}}-v \sin \psi_{\mathrm{e}}+u_{\mathrm{e}} \cos \psi_{\mathrm{e}} \\
& \dot{n}_{\mathrm{e}}=-r_{\mathrm{p}} \tau_{\mathrm{e}}+u_{\mathrm{d}} \sin \psi_{\mathrm{e}}+v \cos \psi_{\mathrm{e}}+u_{\mathrm{e}} \sin \psi_{\mathrm{e}}
\end{aligned}
$$

为了避免侧滑角的二次导数中包含推力 $X$ 的导 数, 以及前向速度为 0 时侧滑角无定义的问题, 这 里采用期望前向速度 $u_{\mathrm{d}}$ 定义侧滑角

$$
\beta_{\mathrm{d}}=\arctan \frac{v}{u_{\mathrm{d}}}
$$

于是式 (8) 可写为

$$
\begin{aligned}
& \dot{\tau}_{\mathrm{e}}=-U_{\mathrm{p}}+r_{\mathrm{p}} n_{\mathrm{e}}+U_{\mathrm{d}} \cos \Psi+u_{\mathrm{e}} \cos \psi_{\mathrm{e}} \\
& \dot{n}_{\mathrm{e}}=-r_{\mathrm{p}} \tau_{\mathrm{e}}+U_{\mathrm{d}} \sin \Psi+u_{\mathrm{e}} \sin \psi_{\mathrm{e}}
\end{aligned}
$$

式中

$$
U_{\mathrm{d}}=\sqrt{u_{\mathrm{d}}^{2}+v^{2}}, \quad \Psi=\psi-\psi_{\mathrm{p}}+\beta_{\mathrm{d}}
$$

考虑到 ASV 侧向运动固有的阻尼特性, 假定 侧向速度 $v$ 是有界的, 即 $|v| \leqslant v_{\text {max }}$, 速度指令 $u_{\mathrm{d}}(t)$ 满足 $0<u_{\mathrm{d} \text { min }} \leqslant u_{\mathrm{d}}(t) \leqslant u_{\mathrm{d} \text { max }}$, 因此 $U_{\mathrm{d}} \leqslant\left(u_{\mathrm{d} \max }^{2}+\right.$ $\left.v_{\max }^{2}\right)^{1 / 2},\left|\beta_{\mathrm{d}}\right| \leqslant \arctan \frac{v_{\max }}{u_{\mathrm{d} \min }}$.

分析路径跟踪误差模型 (10), 切向跟踪误差 $\tau_{\mathrm{e}}$ 可以通过选择适当的 $U_{\mathrm{p}}$ 进行控制, 而法向跟踪误差 $n_{\mathrm{e}}$ 则需通过 $\Psi$ 角进行稳定. 选择期望的 $\Psi$ 为

$$
\Psi_{\mathrm{d}}=-\arctan \left(k_{\mathrm{n}} n_{\mathrm{e}}\right), \quad \Psi_{\mathrm{d}} \in\left(-\frac{\pi}{2}, \frac{\pi}{2}\right)
$$

式中, $k_{\mathrm{n}}>0$ 为法向跟踪控制参数. 定义跟踪误差

$$
\Psi_{\mathrm{e}}=\Psi-\Psi_{\mathrm{d}}
$$

相应的角速率为

$$
\begin{aligned}
& \Omega=\dot{\Psi}=r-r_{\mathrm{p}}+\dot{\beta}_{\mathrm{d}} \\
& \Omega_{\mathrm{e}}=\dot{\Psi}_{\mathrm{e}}=\Omega-\dot{\Psi}_{\mathrm{d}}=r-r_{\mathrm{p}}+\dot{\beta}_{\mathrm{d}}-\dot{\Psi}_{\mathrm{d}}
\end{aligned}
$$

将 $\Psi=\Psi_{\mathrm{e}}+\Psi_{\mathrm{d}}$ 代入式 (10), 并整理为如下的级 联系统形式

$$
\begin{aligned}
\Sigma_{1}:\left[\begin{array}{c}
\dot{\tau}_{\mathrm{e}} \\
\dot{n}_{\mathrm{e}}
\end{array}\right]= & {\left[\begin{array}{c}
-U_{\mathrm{p}}+r_{\mathrm{p}} n_{\mathrm{e}}+U_{\mathrm{d}} \cos \Psi_{\mathrm{d}} \\
-r_{\mathrm{p}} \tau_{\mathrm{e}}+U_{\mathrm{d}} \sin \Psi_{\mathrm{d}}
\end{array}\right]+} \\
& {\left[\begin{array}{cc}
U_{\mathrm{d}} \eta_{\mathrm{s}}\left(\Psi_{\mathrm{d}}, \Psi_{\mathrm{e}}\right) & \cos \psi_{\mathrm{e}} \\
U_{\mathrm{d}} \eta_{\mathrm{c}}\left(\Psi_{\mathrm{d}}, \Psi_{\mathrm{e}}\right) & \sin \psi_{\mathrm{e}}
\end{array}\right]\left[\begin{array}{c}
\Psi_{\mathrm{e}} \\
u_{\mathrm{e}}
\end{array}\right] }
\end{aligned}
$$

$$
\begin{aligned}
\Sigma_{2}: & {\left[\begin{array}{c}
\dot{\Psi}_{\mathrm{e}} \\
\dot{\Omega}_{\mathrm{e}} \\
\dot{u}_{\mathrm{e}}
\end{array}\right] } \\
= & {\left[\begin{array}{c}
\frac{m_{11}-m_{22}}{m_{33}} u v-\frac{d_{33}}{m_{33}} r+\frac{1}{m_{33}} N-\dot{r}_{\mathrm{p}}+\ddot{\beta}_{\mathrm{d}}-\ddot{\Psi}_{\mathrm{d}} \\
\frac{m_{22}}{m_{11}} v r-\frac{d_{11}}{m_{11}} u+\frac{1}{m_{11}} X-\dot{u}_{\mathrm{d}}
\end{array}\right] }
\end{aligned}
$$

式中，

$$
\begin{aligned}
& \eta_{\mathrm{s}}\left(\Psi_{\mathrm{d}}, \Psi_{\mathrm{e}}\right)= \begin{cases}\frac{\cos \Psi-\cos \Psi_{\mathrm{d}}}{\Psi_{\mathrm{e}}}, & \Psi_{\mathrm{e}} \neq 0 \\
0, & \Psi_{\mathrm{e}}=0\end{cases} \\
& \eta_{\mathrm{c}}\left(\Psi_{\mathrm{d}}, \Psi_{\mathrm{e}}\right)= \begin{cases}\frac{\sin \Psi-\sin \Psi_{\mathrm{d}}}{\Psi_{\mathrm{e}}}, & \Psi_{\mathrm{e}} \neq 0 \\
1, & \Psi_{\mathrm{e}}=0\end{cases}
\end{aligned}
$$

系统 $\Sigma_{1}$ 是名义系统 $\Sigma_{1, n}$ 受到系统 $\Sigma_{2}$ 的扰动.

$$
\Sigma_{1, n}:\left[\begin{array}{c}
\dot{\tau}_{\mathrm{e}} \\
\dot{n}_{\mathrm{e}}
\end{array}\right]=\left[\begin{array}{c}
-U_{\mathrm{p}}+r_{\mathrm{p}} n_{\mathrm{e}}+U_{\mathrm{d}} \cos \Psi_{\mathrm{d}} \\
-r_{\mathrm{p}} \tau_{\mathrm{e}}+U_{\mathrm{d}} \sin \Psi_{\mathrm{d}}
\end{array}\right]
$$

首先研究名义系统 $\Sigma_{1, n}$ 的稳定性, 定义李亚普 诺夫函数

$$
V=\frac{1}{2}\left(\tau_{\mathrm{e}}^{2}+n_{\mathrm{e}}^{2}\right)
$$

其导数为

$$
\begin{aligned}
\dot{V}= & -\tau_{\mathrm{e}}\left(U_{\mathrm{p}}-U_{\mathrm{d}}\right)+\tau_{\mathrm{e}} U_{\mathrm{d}}\left(\cos \Psi_{\mathrm{d}}-1\right)+ \\
& n_{\mathrm{e}} U_{\mathrm{d}} \sin \Psi_{\mathrm{d}}
\end{aligned}
$$

选择参考点速度

$$
U_{\mathrm{p}}=U_{\mathrm{d}}+k_{\tau} \tau_{\mathrm{e}}
$$

并考虑到

$$
\sin \Psi_{\mathrm{d}}=-\frac{k_{\mathrm{n}} n_{\mathrm{e}}}{\sqrt{1+\left(k_{\mathrm{n}} n_{\mathrm{e}}\right)^{2}}}, \quad\left|\cos \Psi_{\mathrm{d}}-1\right| \leqslant\left|\sin \Psi_{\mathrm{d}}\right|
$$

有

$$
\dot{V} \leqslant-k_{\tau} \tau_{\mathrm{e}}^{2}+U_{\mathrm{d}} k_{\mathrm{n}} \frac{\left|\tau_{\mathrm{e}}\right|\left|n_{\mathrm{e}}\right|}{\sqrt{1+\left(k_{\mathrm{n}} n_{\mathrm{e}}\right)^{2}}}-\frac{U_{\mathrm{d}} k_{\mathrm{n}} n_{\mathrm{e}}^{2}}{\sqrt{1+\left(k_{\mathrm{n}} n_{\mathrm{e}}\right)^{2}}}
$$

当控制参数满足

$$
k_{\tau}>U_{\mathrm{d}} k_{\mathrm{n}}
$$


时, 有

$$
\begin{aligned}
& -\frac{k_{\tau}}{2} \tau_{\mathrm{e}}^{2}-\frac{U_{\mathrm{d}} k_{\mathrm{n}}}{2}\left(\frac{n_{\mathrm{e}}}{\sqrt{1+\left(k_{\mathrm{n}} n_{\mathrm{e}}\right)^{2}}}\right)^{2}+ \\
& U_{\mathrm{d}} k_{\mathrm{n}} \frac{\left|\tau_{\mathrm{e}}\right|\left|n_{\mathrm{e}}\right|}{\sqrt{1+\left(k_{\mathrm{n}} n_{\mathrm{e}}\right)^{2}}} \leqslant 0 \\
& \dot{V} \leqslant-\frac{k_{\tau}}{2} \tau_{\mathrm{e}}^{2}-\frac{U_{\mathrm{d}} k_{\mathrm{n}}}{2 \sqrt{1+\left(k_{\mathrm{n}} n_{\mathrm{e}}\right)^{2}}} n_{\mathrm{e}}^{2} \leqslant 0
\end{aligned}
$$

于是得到下面的定理.

定理 1: 选择参考点速度式 (21), 控制参数 $k_{\mathrm{n}} 、 k_{\tau}$ 满足不等式 (23) 时, 名义系统 $\Sigma_{1, n}$ 是全局一致渐近 稳定的并且是局部指数稳定的, 即全局 $\mathcal{K}$ 指数稳定 的.

证明: 由式 (19) 定义的李亚普诺夫函数 $V$ 正定 且径向无界, 而其导数 $\dot{V}$ 负定, 因此系统 $\Sigma_{1, n}$ 全局 一致渐近稳定.

对于任意 $l>0$ 在球域

$$
B_{l}=\left\{\left(\tau_{\mathrm{e}}, n_{\mathrm{e}}\right) \in \mathbb{R}^{2}, \sqrt{\tau_{\mathrm{e}}^{2}+n_{\mathrm{e}}^{2}}<l\right\}
$$

内, 有

$$
\dot{V} \leqslant-\frac{k_{\tau}}{2} \tau_{\mathrm{e}}^{2}-\frac{U_{\mathrm{d}} k_{\mathrm{n}}}{2 \sqrt{1+\left(k_{\mathrm{n}} l\right)^{2}}} n_{\mathrm{e}}^{2}
$$

系统 $\Sigma_{1, n}$ 是局部指数稳定的.

因此, 系统 (18) 是全局 $\mathcal{K}$ 指数稳定的.

注意到式 (21) 与文 [3-4] 的控制律相比, 参考点 速度 $U_{\mathrm{p}}$ 中不含 $\cos \Psi_{\mathrm{d}}$ 项, 从而简化了控制方程, 避 免了速度指令与法向跟踪误差的耦合.

考虑系统 $\Sigma_{2}$, 采用反馈线性化方法选择控制

$$
\begin{aligned}
X= & m_{11}\left(-k_{\mathrm{u}} u_{\mathrm{e}}+\dot{u}_{\mathrm{d}}\right)-m_{22} v r+d_{11} u \\
N= & m_{33}\left(-k_{\mathrm{R}} \Omega_{\mathrm{e}}-k_{\Psi} \Psi_{\mathrm{e}}+\dot{r}_{\mathrm{p}}-\ddot{\beta}_{\mathrm{d}}+\ddot{\Psi}_{\mathrm{d}}\right)- \\
& \left(m_{11}-m_{22}\right) u v+d_{33} r
\end{aligned}
$$

式中, $k_{\mathrm{u}}>0 、 k_{\mathrm{R}}>0 、 k_{\Psi}>0$ 为控制参数. 容易得 到, 在式 (28)、(29) 的控制下, 系统 $\Sigma_{2}$ 全局一致指 数稳定.

定理 2: 在式 (12)、(21)、(28) 和 (29) 控制下, 控 制参数 $k_{\mathrm{n}} 、 k_{\tau}$ 满足不等式 (23) 时, ASV 路径跟踪系 统 $\left\{\Sigma_{1}, \Sigma_{2}\right\}$ 是全局 $\mathcal{K}$ 指数稳定的.

证明: 应用文 [8] 中的定理 2.8 和引理 2.9, 检验 其中的假设条件.

(1) 根据定理 1 , 名义系统 $\Sigma_{1, n}$ 是全局 $\mathcal{K}$ 指数稳 定的, 并且选择 $c \geqslant 2 、 \eta \geqslant 0$ 时, 李亚普诺夫函数式 (19) 及其导数满足定理中的假设条件 A1.
(2) 关联项

$$
\boldsymbol{g}=\left[\begin{array}{cc}
U_{\mathrm{d}} \eta_{\mathrm{s}}\left(\Psi_{\mathrm{d}}, \Psi_{\mathrm{e}}\right) & \cos \psi_{\mathrm{e}} \\
U_{\mathrm{d}} \eta_{\mathrm{c}}\left(\Psi_{\mathrm{d}}, \Psi_{\mathrm{e}}\right) & \sin \psi_{\mathrm{e}}
\end{array}\right]
$$

显然有

$$
\|\boldsymbol{g}\| \leqslant \sqrt{2}\left(1+U_{\mathrm{d}}\right)
$$

满足定理中的假设条件 $\mathrm{A} 2$.

(3) 在式 (28)、(29) 的控制下系统 $\Sigma_{2}$ 是全局一 致指数稳定的, 定理中的假设条件 A3 满足.

根据文 [8] 中的定理 2.8 和引理 2.9 , 系统 $\left\{\Sigma_{1}, \Sigma_{2}\right\}$ 是全局 $\mathcal{K}$ 指数稳定的.

\section{4 仿真研究 (Simulation studies)}

为了验证本文提出的 ASV 非线性路径跟踪控 制算法的有效性, 采用挪威科技大学试验模型船 Cybership II ${ }^{[9]}$ 的参数进行仿真实验, 具体参数见表 1 , 并令 $T_{3}=\delta_{1}=\delta_{2}=0$, 即仅依靠尾部 2 台推进器 进行运动控制.

表 1 Cybership II 模型参数

Tab.1 Parameters of Cybership II model

\begin{tabular}{ccc|ccc}
\hline 参数 & 值 & 单位 & 参数 & 值 & 单位 \\
\hline$m$ & 23.8 & $\mathrm{~kg}$ & $N_{\dot{r}}$ & -1.0 & $\mathrm{~kg} \cdot \mathrm{m}^{2}$ \\
$I_{z z}$ & 1.76 & $\mathrm{~kg} \cdot \mathrm{m}^{2}$ & $X_{u}$ & -2.0 & $\mathrm{~kg} / \mathrm{s}$ \\
$X_{\dot{u}}$ & -2.00 & $\mathrm{~kg}$ & $Y_{v}$ & -7.0 & $\mathrm{~kg} / \mathrm{s}$ \\
$Y_{\dot{v}}$ & -10.0 & $\mathrm{~kg}$ & $N_{r}$ & -0.5 & $\mathrm{~kg} \cdot \mathrm{m}^{2} / \mathrm{s}$ \\
\hline
\end{tabular}

船尾推进器产生的推力分别为 $T_{1} 、 T_{2}$, 假设每 台推进器的最大推力为 $50 \mathrm{~N}, L_{y T_{1}}=-0.075 \mathrm{~m}, L_{y T_{2}}=$ $0.075 \mathrm{~m}$ 为推进器安装位置, $T_{1} 、 T_{2}$ 与前向推力 $X$ 和 偏航力矩 $N$ 之间的关系为

$$
\left[\begin{array}{l}
X \\
N
\end{array}\right]=\left[\begin{array}{cc}
1 & 1 \\
L_{y T_{1}} & L_{y T_{2}}
\end{array}\right]\left[\begin{array}{c}
T_{1} \\
T_{2}
\end{array}\right]
$$

选择控制参数 $k_{\mathrm{n}}=0.1, k_{\tau}=0.5, u_{\mathrm{d}}=1 \mathrm{~m} / \mathrm{s}, k_{\mathrm{R}}=5$, $k_{\Psi}=5, k_{\mathrm{u}}=0.2, \mathrm{ASV}$ 的初始状态为: $x(0)=0 \mathrm{~m}$, $y(0)=10 \mathrm{~m}, u(0)=v(0)=0 \mathrm{~m} / \mathrm{s}, r(0)=0 \mathrm{rad} / \mathrm{s}, \psi(0)=$ $\pi$.

\section{1 直线路径跟踪}

以直线长度为曲线参数, 直线路径可以表示为

$$
x_{\mathrm{p}}(\varpi)=\varpi \cos \psi_{\mathrm{sf}}, \quad y_{\mathrm{p}}(\varpi)=\varpi \sin \psi_{\mathrm{sf}}
$$

其中, $\psi_{\mathrm{sf}}=\pi / 4$ 为直线路径与 $x$ 轴的夹角. 仿真结 果如图 2 所示.

\section{2 圆路径跟踪}

以相对 $x$ 轴的旋转角度为曲线参数, 圆路径可 


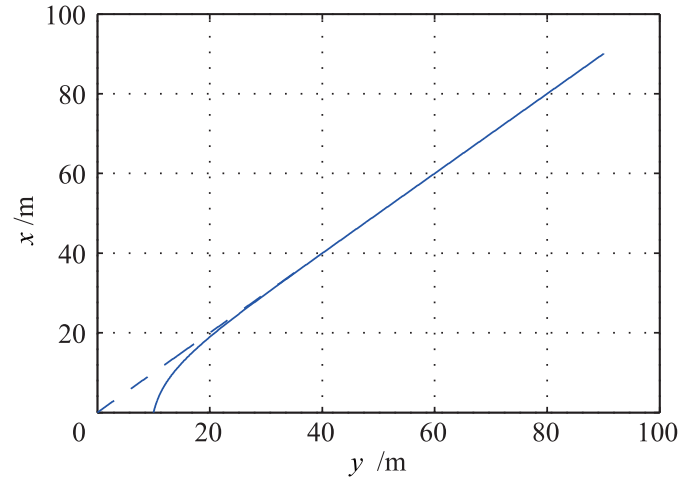

(a) ASV参考路径和仿真轨迹

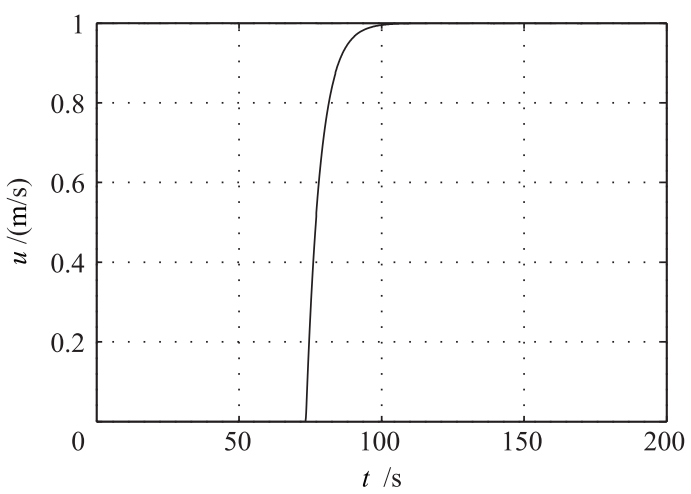

(c) ASV前向速度
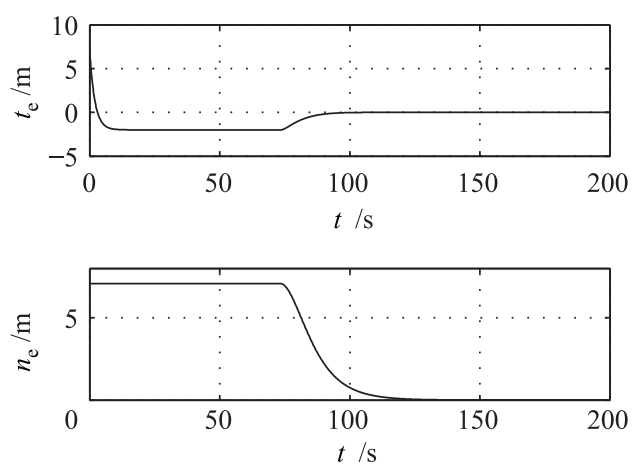

(b) ASV路径跟踪误差
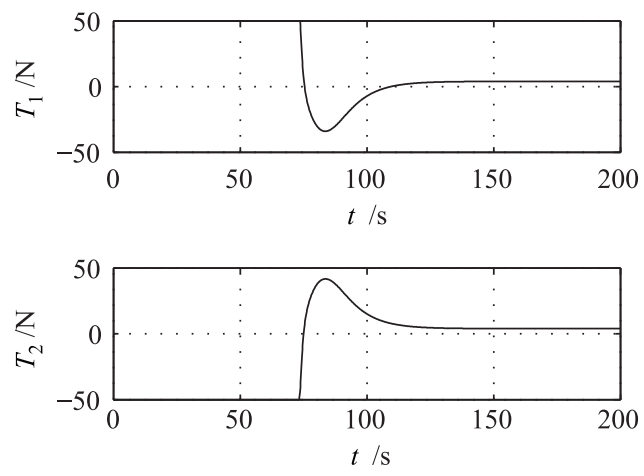

(d) ASV推力

图 $2 \mathrm{ASV}$ 直线路径跟踪曲线

Fig.2 Straight path following results of the ASV

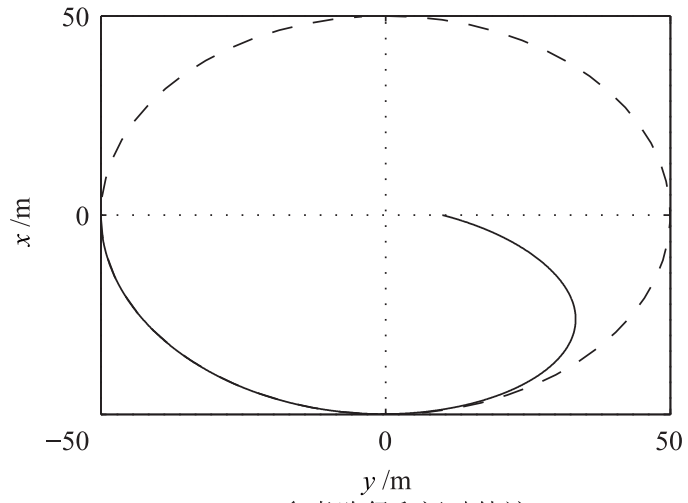

(a) ASV参考路径和运动轨迹

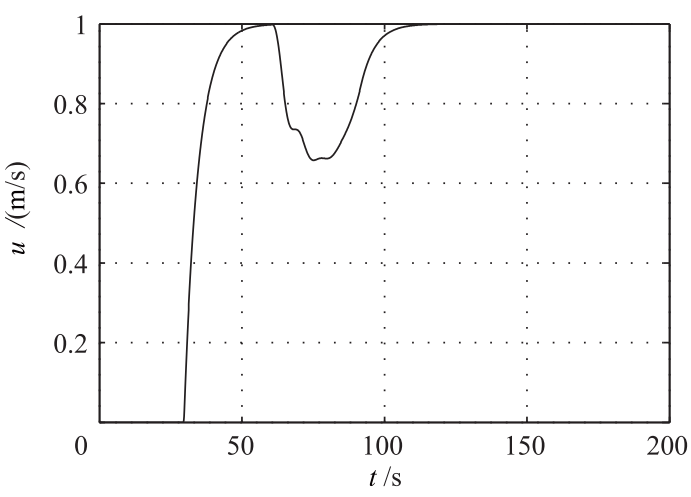

(c) ASV前向速度
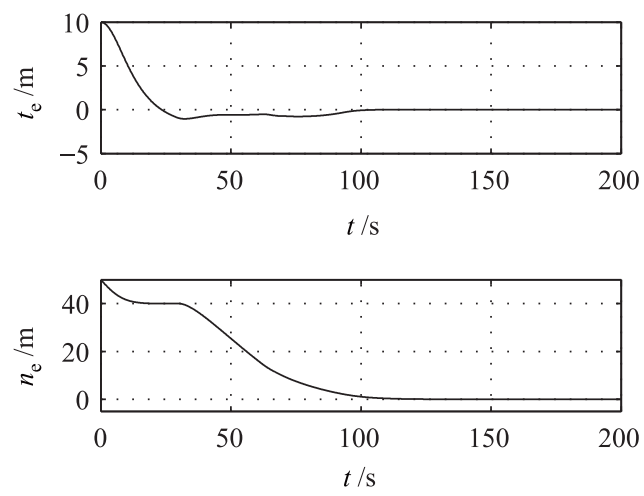

(b) ASV路径跟踪误差
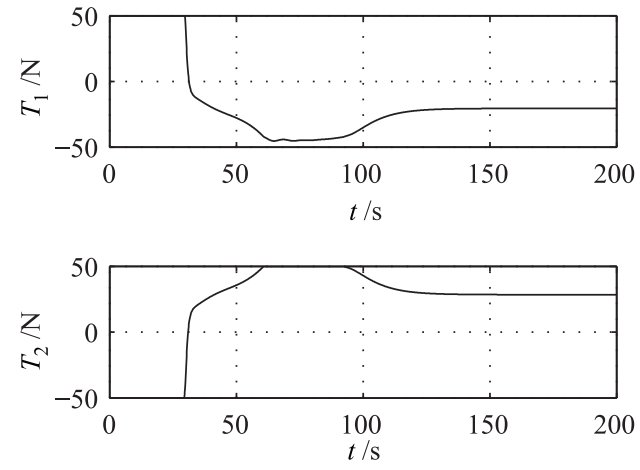

(d) ASV推力

图 $3 \mathrm{ASV}$ 圆路径跟踪曲线

Fig.3 Circle path following results of the ASV 
以表示为

$$
x_{\mathrm{p}}(\varpi)=R \cos \varpi, y_{\mathrm{p}}(\varpi)=R \sin \varpi
$$

其中, $R=50 \mathrm{~m}$ 为圆半径. 仿真结果如图 3 所示.

从上面的仿真结果看到, 本文所提出的路径跟 踪控制算法很好地实现了欠驱动 ASV 的路径跟踪, 跟踪误差 $\tau_{\mathrm{e}} 、 n_{\mathrm{e}}$ 收玫到 0 , 同时保证了前向速度的跟 踪误差收敛到 0 .

从切向跟踪误差曲线可以看到, 在初始位置时 跟踪参考点并不在 ASV 的正交投影点上, 通过对路 径参数 $\varpi$ 的控制, 参考点很快收玫到投影点上. 在 此过程中, 由于推进器的饱和特性, 2 台推进器分 别以 $50 \mathrm{~N}$ 和 $-50 \mathrm{~N}$ 的推力产生最大偏航力矩以跟 踪航向角指令, 而推力 $X$ 为 0 , 前向速度 $u$ 保持为 $0, \mathrm{ASV}$ 的位置不变, 跟踪误差 $\tau_{\mathrm{e}} 、 n_{\mathrm{e}}$ 达到稳定, 直 到 ASV 的速度大于 0 后再逐渐收玫到 0 . 在 ASV 前 向速度为 0 时, 控制律中的侧滑角仍有定义, 不会 出现采用传统侧滑角定义时的奇异值.

在直线路径和圆路径跟踪中, 当系统稳定后, ASV 的速度向量与路径曲线相切, 航向角速度和侧 向速度稳定, 因此, 参考点的运动速度也趋于常值, 等于 ASV 速度向量的幅值.

\section{5 实验结果 (Experimental results)}

为了进一步验证 ASV 路径跟踪控制方法的有 效性和控制性能, 开发了双推进器 ASV 并开展了路 径跟踪控制实验, 如图 4 所示.

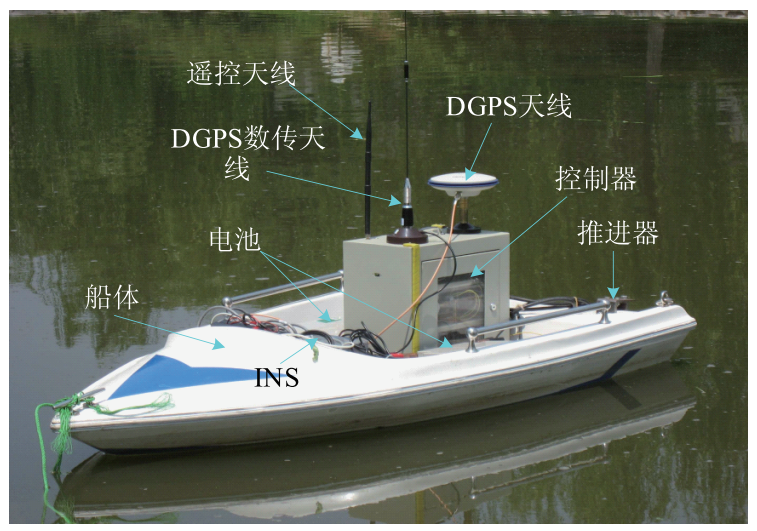

图 4 ASV 实验船

Fig.4 Experimental ASV

ASV 采用实时无线遥控控制方式, 其控制结构 如图 5 所示. ASV 控制器采用 PC104 嵌入式计算机, 控制软件采用 VxWorks 实时操作系统, 以 $200 \mathrm{~ms}$ 为 周期定时接收和处理 INS/DGPS 组合导航系统数据, 并将运动状态信息通过通信模块发送到岸上运动控 制计算机。
岸上运动控制计算机采用安装无线通信模块的 便携式计算机，软件在 Visual Studio 2008 下开发， 接收到 ASV 的状态信息后启动一次控制周期, 完成 路径跟踪控制的解算, 并将推进器指令通过无线通 信下达到 ASV 控制器, 后者将该指令通过 DA 转换 输出为电压信号, 驱动两台尾部推进器转动, 从而 实现 ASV 的运动控制.

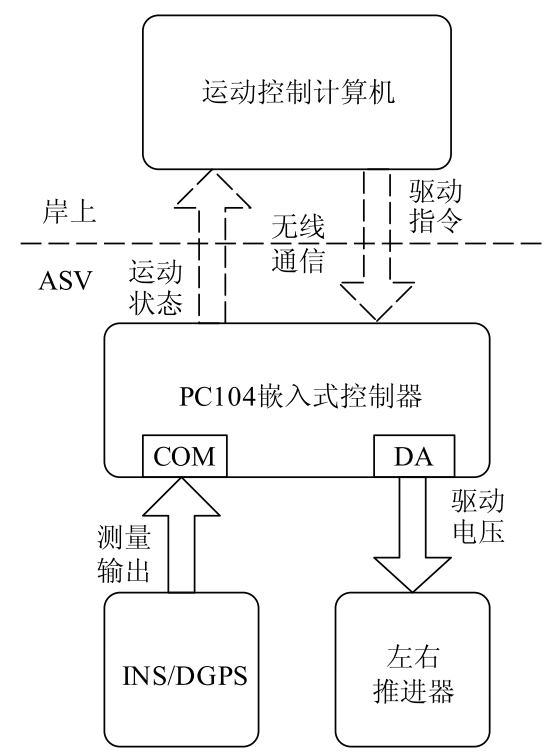

图 5 ASV 控制系统结构

Fig.5 Control system structure of the ASV

选择与仿真研究中相同的路径跟踪控制参数和 速度指令, 即

$$
k_{\mathrm{n}}=0.1, \quad k_{\tau}=0.5, \quad u_{\mathrm{d}}=1 \mathrm{~m} / \mathrm{s}
$$

由于缺少控制算法 (28)、(29) 中的 ASV 模型参 数, 实验船采用不依赖于模型参数的 PI 航向控制和 速度控制. 为了避免在控制初期较大的误差通过 PI 控制的积分积累造成系统较大的超调甚至振荡, 这 里引入积分系数对积分作用进行限制, 即在误差大 于某一门限时取消积分作用, 控制方程如下:

$$
\begin{aligned}
& N=-k_{\Psi_{\mathrm{p}}} \Psi_{\mathrm{e}}-k_{\Psi_{\mathrm{i}}} \rho_{\Psi_{\mathrm{i}}}\left(\Psi_{\mathrm{e}}\right) \int \Psi_{\mathrm{e}} \mathrm{d} t \\
& X=\bar{d}_{11} u_{\mathrm{d}}-k_{\text {up }} u_{\mathrm{e}}-k_{\text {ui }} \rho_{\text {ui }}\left(u_{\mathrm{e}}\right) \int u_{\mathrm{e}} \mathrm{d} t
\end{aligned}
$$

式中, $k_{\Psi \mathrm{p}} 、 k_{\Psi_{\mathrm{i}}} 、 k_{\mathrm{up}} 、 k_{\mathrm{ui}}$ 为控制参数, $\bar{d}_{11}$ 为阻尼系数 的标称值, 积分系数 $\rho_{\Psi \mathrm{i}} 、 \rho_{\mathrm{ui}}$ 定义为

$\rho_{\Psi_{\mathrm{i}}}\left(\Psi_{\mathrm{e}}\right)= \begin{cases}1 & \Psi_{\mathrm{e}} \leqslant 3 \pi / 180 \\ \frac{10 \pi / 180-\Psi_{\mathrm{e}}}{7 \pi / 180} & 3 \pi / 180<\Psi_{\mathrm{e}}<10 \pi / 180 \\ 0 & \Psi_{\mathrm{e}} \geqslant 10 \pi / 180\end{cases}$ 


$$
\rho_{\text {ui }}\left(u_{\mathrm{e}}\right)= \begin{cases}1 & u_{\mathrm{e}} \leqslant 0.1 \\ \frac{0.5-u_{\mathrm{e}}}{0.4} & 0.1<u_{\mathrm{e}}<0.5 \\ 0 & u_{\mathrm{e}} \geqslant 0.5\end{cases}
$$

并通过推力分配以及电压与推力的比例关系得到两 台推进器的驱动电压 $v_{T_{1}} 、 v_{T_{2}}$, 这里限制驱动电压范 围为 $0 \mathrm{~V} \sim 5 \mathrm{~V}$.

在西北工业大学启真湖进行了 ASV 椭圆路径 跟踪控制实验, 以相对 $x$ 轴的旋转角度为路径参数, 椭圆路径方程为

$$
\begin{aligned}
& x_{\mathrm{p}}(\varpi)=15 \cos \varpi+30 \\
& y_{\mathrm{p}}(\varpi)=10 \sin \varpi
\end{aligned}
$$

实验结果如图 6 所示.

从 ASV 湖上实验结果可以看到, 本文提出的路 径跟踪控制具有较高的路径跟踪和速度跟踪精度. 实验中通过参考点控制使切向跟踪误差能够较快 地收玫, 而 ASV 初始的航向与路径切线方向基本 一致, 法向跟踪误差较仿真中收玫得更快, 但存在 $5 \mathrm{~m}$ 左右的超调, 这是由航向跟踪控制中的超调造 成的. 前向速度在 $20 \mathrm{~s}$ 左右稳定到期望速度, 超调 小于 $0.06 \mathrm{~m} / \mathrm{s}$. 与仿真结果相比, ASV 的速度控制有

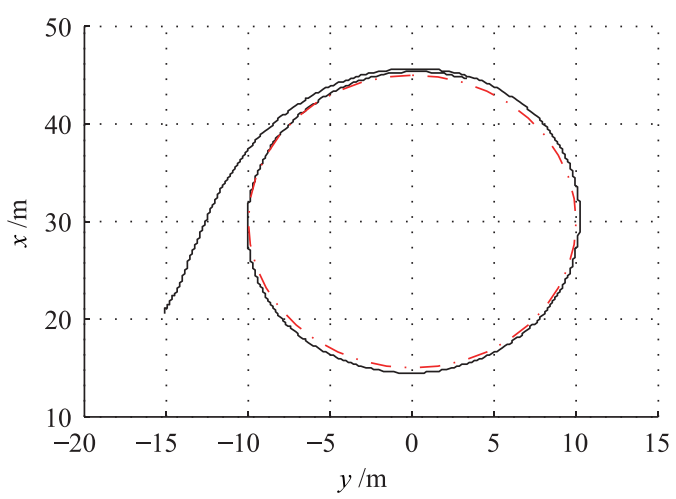

(a) ASV路径跟踪轨迹

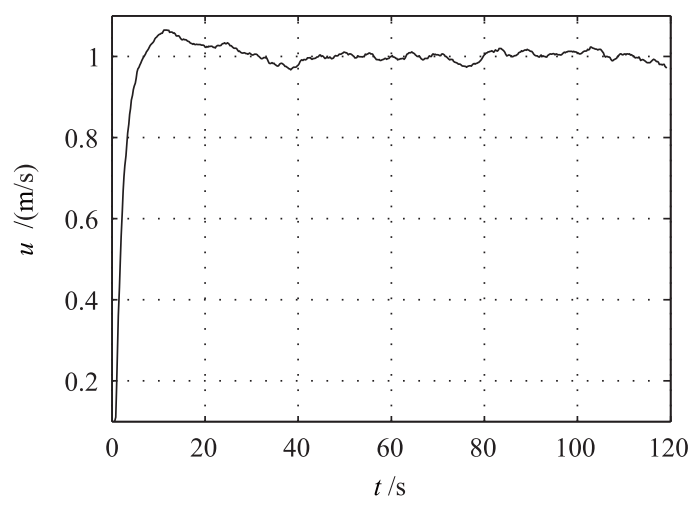

(c) ASV前向速度
小范围的波动, 误差小于 $0.05 \mathrm{~m} / \mathrm{s}$, 分析认为这主要 是湖中的不稳定水流和水面风的干扰造成的. 另外, 卷入螺旋桨的水草也会对控制产生影响.

总的来说, 实验中虽然 ASV 实验船的航向和速 度控制采用了与理论研究所不同的 PI 控制, 但只要 能够很好地跟踪航向角指令和速度指令, 依然能够 保证较高的路径跟踪控制精度和品质.

\section{6 总结 (Conclusions)}

本文研究了欠驱动 ASV 的路径跟踪控制问题, 在以自由路径参考点为原点的 Serret-Frenet 坐标系 下描述路径跟踪误差, 并采用一般曲线参数描述参 考点的坐标及其在参考路径上的运动, 基于级联方 法, 将 ASV 的航向和速度控制与路径跟踪控制分 离, 证明了路径跟踪误差的全局 $\mathcal{K}$ 指数稳定性.

与以往的 ASV 路径跟踪方法不同的是, 本文采 用级联方法进行控制律设计和稳定性证明, 得到的 控制律比其它基于反步法设计的控制律更为简洁, 并获得了全局 $\mathcal{K}$ 指数稳定性. 用期望的前向速度 定义侧滑角, 避免了二阶导数中包含控制量 $X$ 的导 数而难以求解, 以及前向速度为 0 时侧滑角无定义 的问题. 通过对直线和圆参考路径的仿真研究以及
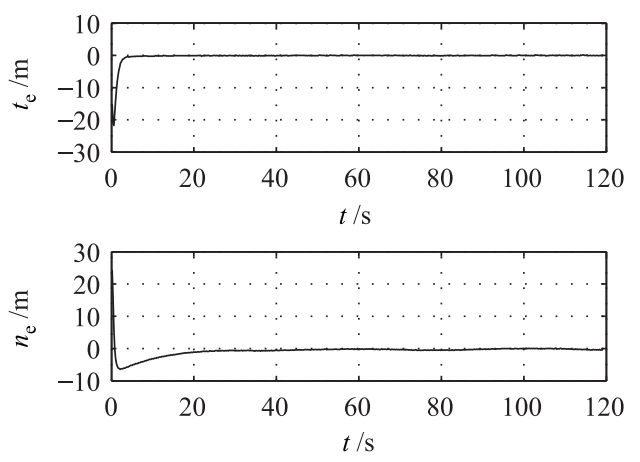

(b) ASV路径跟踪误差
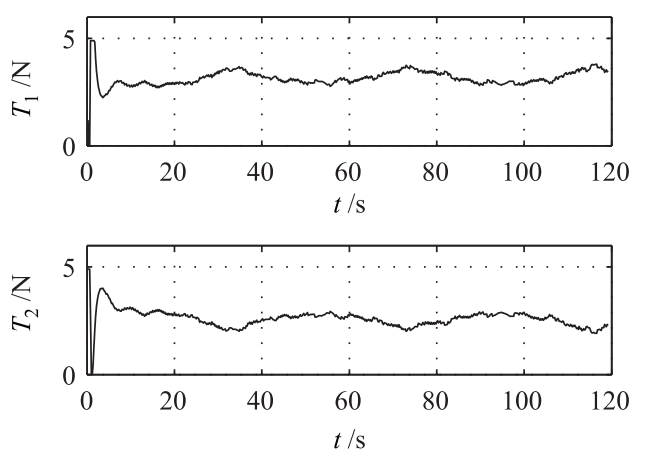

(d) ASV推进器驱动电压

图 $6 \mathrm{ASV}$ 路径跟踪控制实验结果

Fig.6 ASV path following control experiment results 
ASV 湖上实验研究，验证了该路径跟踪控制的精度 和性能.

$$
\text { 下一步将研究水流和风干扰条件下的 ASV 路 }
$$
径跟踪控制问题, 并采用系统辨识算法获得 ASV 的 运动模型参数, 以改善跟踪控制的性能.

\section{参考文献 (References)}

[1] 郭晨, 汪洋, 孙富春, 等. 欠驱动水面船舶运动控制研究综 述 [J]. 控制与决策, 2009, 24(3): 321-329.

Guo C, Wang Y, Sun F C, et al. Survey for motion control of underactuated surface vessels[J]. Control and Decision, 2009, 24(3): 321-329.

[2] Do K D, Pan J. State- and output-feedback robust pathfollowing controllers for underactuated ships using SerretFrenet frame[J]. Ocean Engineering, 2004, 31(5/6): 587-613.

[3] Lapierre L, Soetanto D, Pascoal A. Nonlinear path following with applications to the control of autonomous underwater vehicles[C]//42nd IEEE Conference on Decision and Control. Piscataway, NJ, USA: IEEE, 2003: 1256-1261.

[4] Lapierre L, Jouvencel B. Robust nonlinear path-following control of an AUV[J]. IEEE Journal of Oceanic Engineering, 2008, 33(2): 89-102.

[5] Breivik M, Fossen T I. Guidance laws for planar motion control[C]//47th IEEE Conference on Decision and Control. Piscataway, NJ, USA: IEEE, 2008: 570-577.
[6] Maurya P, Aguiar A P, Pascoal A M. Marine vehicle path following using inner-outer loop control[C]//8th IFAC International Conference on Manoeuvring and Control of Marine Craft. Kidlington, UK: Elsevier Science, 2009.

[7] Panteley E, Loría A. On global uniform asymptotic stability of non linear time-varying systems in cascade[J]. Systems and Control Letters, 1998, 33(2): 131-138.

[8] Loría A, Panteley E. Cascaded nonlinear time-varying systems: Analysis and design[M]//Lecture Notes in Control and Information Sciences, vol.311. Berlin, Germany: Springer-Verlag, 2005.

[9] Fossen Thor I. Marine control systems: Guidance, navigation and control of ships, rigs and underwater vehicles[M]. Trondheim, Norway: Marine Cybernetics, 2002.

[10] Sveen D A. Robust and adaptive tracking control of surface vessel for synchronization with an ROV: Practical implementation on Cybership II[D]. Trondheim, Norway: Norwegian University of Science and Technology, 2003.

\section{作者简介:}

高 剑 (1979-), 男, 博士, 副教授. 研究领域: 水下航行 器运动控制与协同控制.

刘富樯 (1986-), 男, 博士生. 研究领域: 水下航行器协同 控制, 嵌入式控制.

\section{(上接第 328 页)}

[11] Pfaff P, Triebel R, Burgard W. An efficient extension to elevation maps for outdoor terrain mapping and loop closing[J]. International Journal of Robotics Research, 2007, 26(2): 217-230.

[12] Rivadeneyra C, Miller I, Schoenberg J R, et al. Probabilistic estimation of multi-level terrain maps[C]//IEEE International Conference on Robotics and Automation. Piscataway, NJ, USA: IEEE, 2009: 1643-1648.

[13] Orghidan R, Mouaddib E M, Salvi J, et al. Catadioptric singleshot rangefinder for textured map building in robot navigation[J]. IET Computer Vision, 2007, 1(2): 43-53.

[14] Olson C F, Matthies L H, Wright J R, et, al. Visual terrain mapping for Mars exploration[J]. Computer Vision and Image Understanding, 2007, 105(1): 73-85.

[15] Miller I, Campbell M. A mixture-model based algorithm for real-time terrain estimation[J]. Journal of Field Robotics, 2006, 23(9): 755-775.
[16] Kleiner A, Dornhege C. Real-time localization and elevation mapping within urban search and rescue scenarios[J]. Journal of Field Robotics, 2007, 24(8/9): 723-745.

[17] Vandenberghe L, Boyd S. Semidefinite programming[J]. SIAM Review, 1996, 38(1): 49-95.

[18] Bar-Shalom Y, Li Y R, Kirubarajan T. Estimation with applications to tracking and navigation: Theory, algorithm and software[M]. New York, NY, USA: Wiley, 2001.

\section{作者简介:}

周 波 (1981-), 男, 博士, 讲师. 研究领域: 非线性估计 与控制, 机器人技术.

戴先中（1954-)，男，博士，教授. 研究领域: 非线性系统 理论, 电力系统控制, 机器人技术和应用.

韩建达 (1968-), 男, 博士, 研究员. 研究领域: 机器人控 制, 多机器人系统, 智能控制系统设计. 